\title{
Prevalence of enteroviruses in children with and without hand, foot, and mouth disease in China
}

\author{
Xiaoai Zhang ${ }^{2+}$, Hongyu Wang ${ }^{1,2+}$, Shujun Ding ${ }^{3}$, Xianjun Wang ${ }^{3}$, Xiaodan Chen², Ying Wo ${ }^{2}$, Liyuan Wang ${ }^{1,2}$, \\ Doudou Huang ${ }^{1,2}$, Wei Liu ${ }^{2 *}$ and Wuchun CaO ${ }^{2^{*}}$
}

\begin{abstract}
Background: To determine the prevalence of human enteroviruses (HEVs) among healthy children, their parents, and children with hand, foot, and mouth disease (HFMD).

Methods: We conducted a case-control study that included throat samples from 579 children with HFMD and from 254 healthy controls. Throat samples from 49 households (98 parents and 53 healthy children) were also analyzed. Phylogenetic analysis was carried out to study genetic relationships of EV71 strains.

Results: The HEV positive rate in HFMD patients was significantly higher than that in healthy controls $(76.0 \% \mathrm{vs}$. $23.2 \%, P<0.001)$. The EV71 (43.7\% vs. $15.0 \%, P<0.001)$, CVA16 (18.0\% vs. $2.8 \%, P<0.001)$, and CVA10 (5.7\% vs. $0.8 \%$, $P=0.001)$ serotypes were significantly overrepresented in HFMD patients in comparison to healthy children. Other HEV serotypes were detected with comparable frequency in cases and controls. The HEV positive rate in severe HFMD patients was significantly higher than that in mild group ( $82.1 \%$ vs. $73.8 \%, P=0.04)$. The EV71 (55.0\% vs. $39.7 \%, P=0.001)$ and CVA16 (11. 9\% vs. 20.0\%, $P=0.024)$ positive rate differed significantly between severe and mild HFMD patients. Other HEV serotypes were detected with comparable frequency between severe and mild HFMD patients. Among 49 households, 22 households (44.9\%) had at least 1 family member positive for HEV. Children had significantly higher HEV positive rate than adult ( $28.3 \%$ vs. $14.3 \%, P=0.037)$. The HEV positive rate was similar between mothers and fathers (12.24\% vs. 16.32\%, $P=0.56)$. The VP1 sequences of EV71 from HFMD patients and healthy children were nearly identical and all were clustered in the same clade, C4a.
\end{abstract}

Conclusions: Our study demonstrated the co-circulation of multiple HEV serotypes in children with and without HFMD during epidemic. Our study deserves the attention on HFMD control.

Keywords: Hand, foot, and mouth disease, Enterovirus, Epidemiology

\section{Background}

Enteroviruses (EVs) are among the most common human viruses infecting humans, causing a wide spectrum of illness. On the basis of phylogenetic analysis, the genus Enterovirus (family Picornaviridae) is divided into 12 species (www.picornaviridae.com). Members of human enteroviruses (HEVs) include 7 species, four HEV species and 3 recently subsumed human rhinoviruses species. Although infections caused by HEVs are often asymptomatic or mild, they can cause more severe conditions, such as neurological

\footnotetext{
*Correspondence: liu_weis@sohu.com; caowc@bmi.ac.cn

${ }^{\dagger}$ Equal contributors

${ }^{2}$ State Key Laboratory of Pathogen and Biosecurity, Beijing Institute of Microbiology and Epidemiology, 20 Dong-Da Street, Fengtai District, Beijing 100071, P. R. China

Full list of author information is available at the end of the article
}

disease, poliomyelitis, severe neonatal systemic disease, encephalitis, meningitis, or myocarditis.

Hand, foot, and mouth disease (HFMD) is a common disease caused by HEV infection among children, particularly in those less than 5-year-old. HFMD occurs worldwide epidemically, with enterovirus 71 (EV71) and coxsackievirus A16 (CVA16) taking predominant roles in causing outbreak, while other HEV serotypes were largely associated with sporadic cases. In the past decade, the size and frequency of HFMD outbreaks have greatly increased in the Asia-Pacific region, especially in Southeast Asia [1,2]. In China, a large scale outbreak of HFMD emerged in 2007 in Shandong Province, with 1149 cases reported [3]. The nationwide epidemics of HFMD started in 2008 in Anhui province, with approximately 490,000 cases reported 
[4]. Since then, there has been a large outbreak of HFMD annually in China. In 2012, the Chinese Center for Disease Control and Prevention (China CDC) confirmed 2,168,737 cases in Mainland China including 569 deaths (published on the website of the Ministry of Health of China). HFMD has become an important public health issue in China.

Since HFMD was classified as a category $C$ notifiable infectious disease by the Ministry of Health of China in 2008, the laboratory detection of EV71 and CVA16 has been routinely performed in pediatric sentinel sites set by the Infectious Disease Surveillance Center for HFMD patients. This allowed an extensive epidemiological and genetic characterization of the EV71 and CVA16 infection nationwide. However, the negative detection of EV71 and CVA16 had been reported frequently in China [5-7]. In few studies, other HEV serotypes were investigated, and suggested that CVA10, CVA6, CVA4 and CVA12 were associated with sporadic HFMD cases [6,7]. However, none of the aforementioned studies could ascertain their causal associations with HFMD development or disease severity of HFMD; due to lack of a four-fold antibody titer increase in convalescent samples, or make any comparison with healthy subjects. In addition, most previous data on HEV circulation have been reported from analysis of specimens from patients $[7,8]$, whereas little epidemiologic information is available for the HEV circulation in healthy population, especially in China [9-11]. To address this query, a designed case-control study was performed to identify the HEV circulation in children with and without HFMD. Household distribution of HEVs was also investigated to disclose the epidemiologic characteristics of household distribution of HEVs in the healthy population.

\section{Methods}

\section{Sample collection}

The case-control study was performed in Jining city in Shandong province from May to October 2010. The children diagnosed as HFMD in Jining city were recruited into the study in the sentinel hospitals set by the national surveillance program for HFMD in Shandong province. The patients were identified according to the diagnostic criteria defined by the Ministry of Health (http://www.moh.gov.cn/publicfiles/business/htmlfiles/ mohyzs/s3586/201004/46884.htm). Children with serious complications, including encephalitis, meningitis, acute flaccid paralysis, cardiorespiratory failure of death, were considered as severe HFMD. Children diagnosed as HFMD, but without any of the above mentioned serious complications, were classified as mild HFMD. Medical records of the HFMD patients were reviewed by physicians to collect the demographic data, the clinical symptoms and signs, laboratory findings, clinical diagnoses and outcomes.

Healthy children in Jining city were recruited as controls during the same period when the cases were recruited. Four villages that served as the sources of the patients in the selected hospitals were randomly selected as study sites. In each of two villages, one kindergarten was randomly selected to recruit healthy children less than 5-year-old. In other two villages, healthy children of less than 3-yearold were randomly recruited from families. Throat swabs were collected and personal information on demographic factors and medical history were obtained from their guardians by using a standard questionnaire. Throat swabs from parents who took care of healthy children and were willing to participate into the study in the two villages were collected simultaneously when sampling children. For the parents, personal information on demographic factors and medical history were collected via a questionnaire. All the recruited healthy children and parents did not exhibit HFMD-related symptoms before or at the time of sample collection.

This study was performed with the approval of the Ethical Committee of Beijing Institute of Microbiology and Epidemiology and Jining Infectious hospital and was conducted according to the principles expressed in the Declaration of Helsinki. At recruitment, written informed consent was obtained from all participants or guardians of pediatric participants.

\section{Detection and genotyping of HEV}

For the detection of HEV, RNA were extracted from each specimen by using QIAamp ${ }^{\oplus}$ MinElute Virus Spin Kits (QIAGEN, Hilden, Germany) and the cDNA sample was synthesized by using SuperScript ${ }^{\ominus}$ III First-Strand Synthesis System for Reverse Transcription Polymerase Chain Reaction (RT-PCR) (Invitrogen, America). The detection of HEV and further classification of EV71 and CVA16 for HEV-positive samples were performed by realtime PCR using previously described primers, respectively [12]. To further identify the HEV serotypes other than EV71 and CVA16, semi-nested RT-PCR specific for a 5 ' partial region of VP1 was performed for other HEVpositive samples by using previously reported primers [13]. The amplicons were subjected to sequencing and BLAST analysis.

\section{Sequence analysis of EV71}

The VP1 sequences (891 bp) for EV71 positive samples from healthy children and HFMD patients were amplified by nested-PCR using primers (Table 1). The genomic sequences were assembled using Lasergene's DNA SeqMan software (version 7.1.0, DNA Star Inc. Madison, WI, USA). The sequences obtained from the study were submitted to NCBI with the GenBank Accession Numbers: KF704042KF704050, HQ668342, HQ668360, HQ668388, HQ668400, and HQ668414-HQ668422. The MEGA program (version 5.05, Sudhir Kumar, Arizona State University) was used for alignments and phylogenetic tree construction by 
Table 1 Primers used for enterovirus 71 VP1 sequencing by RT-PCR

\begin{tabular}{llll}
\hline Primer & Sequence & Position & Usage \\
\hline VP1-2382 F1 & 5'-ATAATAGCACTAGCGGCAGCCCA-3' & 2382 & Nested RT-PCR, 1st round \\
VP1-2415 F2 & 5'-ACCATGAAGTTGTGCAAGGA-3' & 2415 & Nested RT-PCR, 2st round \\
VP1-3387R2 & 5'-GCCCCAGACTGTTGTCCAAA-3' & Nested RT-PCR, 2st round \\
VP1-3478R1 & 5'-GTCGCGAGAGCTGTCTTCCCA-3' & 3387 & Nested RT-PCR, 1st round \\
2833F & 5'-GAGYTRTCACCTACATGCG-3' & 3478 & Sequencing \\
3065R & 5'-CTCGCRGGTGACATGAAYGG-3' & 2833 & Sequencing \\
\hline
\end{tabular}

neighbor-joining method or maximum likelihood method with 1000 bootstrap pseudo replicates. Similarities between strains were calculated by using BioEdit (version 7.13, www.mbio.ncsu.eud/bioedit/bioedit.html).

\section{Statistical analysis}

Descriptive statistics were performed, with continuous variables summarized as median and range, and categorical variables summarized as frequencies and proportions. The statistical significance of difference in HEV prevalence rates between various groups was tested using the $t$ test for continuous variables and the $\chi^{2}$ test and Fisher's exact test for categorical data. Analyses were performed using SPSS, version 11.5 (SPSS).

\section{Results}

\section{Prevalence and serotypes of HEV in cases and controls}

Altogether 579 HFMD patients were recruited into the study, with age ranging from 4 to 97 months (median, 27.5 months) and 395 (68.2\%) were males. Among all the tested HFMD patients, 440 (76.0\%) were infected with HEV, among whom the frequency of HEVs in severe HFMD patients was significantly higher than that in mild disease group, 82.1\% (124/151) vs. 73.8\% (316/428) $(P=0.04)$. Among $440 \mathrm{HEV}$ positive samples from patients, 429 were successfully typed and 12 serotypes were identified: EV71 (253, 43.7\%), CVA16 (104, 18.0\%), CVA10 (33, 5.7\%), CVA6 (22, 3.8\%), CVA12 (6, 1.0\%), echovirus 9 (3, 0.5\%), echovirus 6 (2, 0.4\%), CVB6 (2, 0.4\%), CVA4 (1, $0.2 \%)$, CVA14 (1, 0.2\%), echovirus $24(1,0.2 \%)$ and echovirus 3 (1, 0.2\%). Respectively eight and eleven serotypes were determined from severe and mild HFMD patients. The EV71 (55.0\% vs. $39.7 \%, P=0.001)$ and CVA16 (11.9\% vs. $20.0 \%, P=0.024)$ positive rate differed significantly between severe and mild HFMD patients. For the rarely detected serotypes, CVA14 was only detected from severe cases, while echovirus 3, echovirus 24, CVB6 and CVA4 were only detected from mild cases. The frequencies of other five serotypes were evenly distributed between the two groups (all $P>0.05$ ). The detailed distribution of HEV serotypes in HFMD patients is shown in Table 2.

In total, 254 healthy children were included as control and had throat swabs collected. Their age ranged from 3 to 72 months (median: 48.0 month) and 136 (53.5\%) were male. Altogether 59 (23.2\%) of 254 throat swabs were found to be positive for $\mathrm{HEV}$, and $56 \mathrm{HEV}$ positive samples were successfully typed. Eight serotypes were determined with the most frequently presented serotypes as EV71 $(38,15.0 \%)$ and CVA16 (7, 2.8\%), followed by CVA6 (4, 1.6\%), CVA10 (2, 0.8\%), echovirus 3 (2, 0.8\%), echovirus 24 (1, 0.4\%), echovirus 9 (1, 0.4\%), and CVB6 (1, 0.4\%).

In comparison with HFMD patients, the frequencies of HEVs in healthy controls were significantly lower, $76.0 \%$ $(440 / 579)$ vs. $23.2 \%(59 / 254)(P<0.001)$. The EV71 $(43.7 \%$ vs. $15.0 \%, P<0.001)$, CVA16 $(18.0 \%$ vs. $2.8 \%, P<0.001)$, and CVA10 (5.7\% vs. $0.8 \%, P=0.001)$ serotypes were significantly overrepresented in HFMD cases in comparison to healthy controls. The frequencies of other five serotypes (CVA6, echovirus 3, echovirus 9, echovirus 24, CVB6) were not significantly different between the two groups (all $P>0.05$ ). Four HEV serotypes (echovirus 6, CVA12, CVA4 and CVA14) were only detected from HFMD cases, while not from healthy controls.

\section{Prevalence and serotypes of HEV among households}

A total of 49 households involving 98 parents and 53 healthy children were recruited and sampled. The HEV infection rate for all family members was 19.2\% (29/151). Children had significantly higher HEV infection rate than adults (28.3\% vs. $14.3 \%, P=0.037)$. The HEV infection rates were similar between mothers $(12.24 \% ; 6 / 49)$ and fathers (16.32\%; 8/49) $(P=0.56)$. Twenty-two households (44.9\%) had at least 1 family member with evidence of HEV infections, comprising seven serotypes: EV71 (11.92\%), CVA16 (2.65\%), CVA10 (1.32\%), CVA6 (1.32\%), echovirus 9 (0.66\%), echovirus $3(0.66 \%)$, and CVA4 (0.66\%). In four households, both children and parents were infected with HEVs, although the virus serotypes involved were different within each family. The remaining 18 households had only children or parents with evidence of HEV infections. Among them, 10 households had CVA16, CVA6, E3, EV71, CVA10 infections in children, while 8 households had EV71, CVA16, CVA10, and CVA4 infections in parents (Table 3). There were 2 households (household 44 and 45) where both father and mother had HEV infections, and the same HEV serotype (EV71) was detected between father and mother. Among 4 households which had two children, 
Table 2 Prevalences of enterovirus serotypes in children with and without hand, foot, and mouth disease

\begin{tabular}{|c|c|c|c|c|c|}
\hline & \multicolumn{2}{|c|}{ HFMD cases } & \multirow{2}{*}{$\begin{array}{c}\text { Healthy } \\
\text { children }(n=254)\end{array}$} & \multicolumn{2}{|c|}{$P$ value ${ }^{\mathrm{b}}$} \\
\hline & $\begin{array}{l}\text { Severe HFMD } \\
\quad(n=151)\end{array}$ & $\begin{array}{l}\text { Mild HFMD } \\
(\mathrm{n}=428)\end{array}$ & & $\begin{array}{l}\text { Severe HFMD } \\
\text { vs. mild HFMD }\end{array}$ & $\begin{array}{l}\text { HFMD cases vs. } \\
\text { healthy children }\end{array}$ \\
\hline \multicolumn{6}{|l|}{ HEV status } \\
\hline Negative & $27(17.9)$ & $112(26.2)$ & 195 (76.8) & 0.04 & $<0.001$ \\
\hline Positive & $124(82.1)$ & $316(73.8)$ & $59(23.2)$ & & \\
\hline \multicolumn{6}{|c|}{ HEV serotypes ${ }^{a}$} \\
\hline EV71 & $83(55.0)$ & $170(39.7)$ & $38(15.0)$ & 0.001 & $<0.001$ \\
\hline CVA16 & 18 (11.9) & $86(20.0)$ & $7(2.8)$ & 0.024 & $<0.001$ \\
\hline CVA10 & $11(7.3)$ & $22(5.1)$ & $2(0.8)$ & 0.33 & 0.001 \\
\hline CVA6 & $4(2.7)$ & $18(4.2)$ & $4(1.6)$ & 0.39 & 0.09 \\
\hline E3 & 0 & $1(0.2)$ & $2(0.8)$ & ND & 0.17 \\
\hline E9 & $2(1.3)$ & $1(0.2)$ & $1(0.4)$ & 0.11 & 0.81 \\
\hline E24 & 0 & $1(0.2)$ & $1(0.4)$ & ND & 0.55 \\
\hline CVB6 & 0 & $2(0.5)$ & $1(0.4)$ & ND & 0.91 \\
\hline E6 & $1(0.7)$ & $1(0.2)$ & 0 & 0.44 & ND \\
\hline CVA12 & $2(1.3)$ & $4(0.9)$ & 0 & 0.68 & ND \\
\hline CVA4 & 0 & $1(0.2)$ & 0 & ND & ND \\
\hline CVA14 & $1(0.7)$ & 0 & 0 & ND & ND \\
\hline Untyped & $2(1.3)$ & $9(2.1)$ & $3(1.2)$ & ND & ND \\
\hline
\end{tabular}

${ }^{\mathrm{a} H F M D}$, hand, foot, and mouth disease; EV, enterovirus; CV, coxsackievirus; E, echovirus; NA; ND, not determined.

${ }^{b}$ Fisher exact test or $x^{2}$ test.

Table 3 Circulation of enterovirus in healthy family members in Jining city, Shandong Province

\begin{tabular}{|c|c|c|c|c|c|c|c|}
\hline \multirow{2}{*}{$\begin{array}{c}\text { No.(\%) of } \\
\text { families }(n=49)\end{array}$} & \multicolumn{2}{|c|}{ HEV status } & \multicolumn{5}{|c|}{ HEV serotypes ${ }^{a}$} \\
\hline & Children & Parents & Households & Child 1 & Child 2 & Father & Mother \\
\hline \multirow[t]{2}{*}{$27(55.1)$} & Negative & Negative & $1-26$ & Negative & - & Negative & Negative \\
\hline & & & 27 (2 children) & Negative & Negative & Negative & Negative \\
\hline \multirow[t]{2}{*}{$10(20.4)$} & Positive & Negative & $28-36$ & $\begin{array}{c}\operatorname{EV71}(n=3), \operatorname{CVA} 16(n=2), C V A 6 \\
(n=2), \operatorname{CVA10}(n=1), \text { E3 }(n=1),\end{array}$ & - & Negative & Negative \\
\hline & & & 37 (2 children) & EV71 & EV71 & Negative & Negative \\
\hline \multirow[t]{8}{*}{$8(16.3)$} & Negative & Positive & 38 (2 children) & Negative & Negative & EV71 & Negative \\
\hline & & & 39 (2 children) & Negative & Negative & EV71 & Negative \\
\hline & & & 40 & Negative & - & CVA4 & Negative \\
\hline & & & 41 & Negative & - & Negative & EV71 \\
\hline & & & 42 & Negative & - & Negative & EV71 \\
\hline & & & 43 & Negative & - & Negative & EV71 \\
\hline & & & 44 & Negative & - & EV71 & EV71 \\
\hline & & & 45 & Negative & - & EV71 & EV71 \\
\hline \multirow[t]{4}{*}{$4(8.2)$} & Positive & Positive & 46 & EV71 & - & CVA10 & Negative \\
\hline & & & 47 & CVA9 & - & EV71 & Negative \\
\hline & & & 48 & EV71 & - & CVA16 & Negative \\
\hline & & & 49 & EV71 & - & Negative & CVA16 \\
\hline
\end{tabular}

${ }^{a} E V$, enterovirus; CV, coxsackievirus; $E$, echovirus. 
siblings were all infected with EV71 in family 37, and siblings were all negative for HEV in other 3 households (households 27, 38 and 39).

\section{Genetic characterization of EV71 circulating in healthy children and HFMD patients}

Among 38 EV71 positive specimens from healthy children, half $(n=19)$ were randomly selected and 9 entire VP1 sequences were successfully amplified and sequenced. Among 253 EV71 positive specimens from HFMD patients, $10 \%(\mathrm{n}=25)$ were randomly selected and 13 VP1 sequences were successfully amplified and sequenced. The failure to sequence EV71 VP1 in other specimens positive by RT-PCR may be ascribed to low viral load. The phylogenetic tree was constructed with the VP1 nucleotide sequences of both healthy children and HFMD patients from the present study and those downloaded from GenBank (Figure 1). All the sequenced EV71 strains in the current study were classified as belonging to the C4a group, demonstrating highest similarity with strains from Shandong province. The EV71 strains identified from healthy children and HFMD patients in this study showed sequence identity of $97.1 \%-100 \%$. In addition, all isolates from members living in the same household (households 44 and 45 where both husband and wife had EV71 infection, and household 37 with all siblings infected with EV71) had 100\% sequence homology, suggesting a possible interfamilial spread of this virus strain.

\section{Discussion}

In this study, we identified a variety of HEV genotypes from throat swabs of HFMD patients in Jining, China. Our results show a diversified pathogen composition which is similar with reports from other areas of China [7,14-16], Spain [17], Korea [18], India [19] and Singapore [20]. By comparison between HFMD cases and healthy controls, we found that EV71, CVA16, and CVA10 serotypes were more frequently related to HFMD. In contrast, other five serotypes (CVA6, echovirus 3, echovirus 9, echovirus 24, CVB6) were detected in the two groups of subjects with comparable frequency. Some HEV serotypes were only detected from HFMD cases, and not from healthy controls. However, their roles cannot be confirmed due to the small number of positives found. In this study, CVA6 was detected infrequently whereas it has been associated with sporadic HFMD cases and outbreaks occurred recently in Japan [21], Spain [22], USA [23] and Thailand [24]. A study performed in Taiwan in 2010 documented CVA6 as a major cause of atypical HFMD, while CVA16 and EV71 were reported rarely [25]. In addition, we found that EV71 was overrepresented in severe HFMD patients, concurring with previous studies showing extremely high circulations of EV71 in more severe cases [26]. Our results also supported the notion that EV71 and CVA16 circulate widely and actively in China as two main causative agents of HFMD, as previously reported [6,27].

The aetiology of HFMD has changed with time occurred in China and in several other countries [15,16,24]. The sentinel surveillance studies performed in Linyi City, Shandong province, China, showed that the pathogen spectrum changed from 2008 to 2011, with the most prevalent HEV serotype being EV71 between 2008 to 2010, and CVA16 in $2011[4,15,28]$. CVA6 replaced CVA16 as the second most common serotype between 2010 to 2012 in Shenzhen, China [16]. In Thailand, HFMD is usually caused by EV71 and CVA16, but an outbreak of HFMD in 2012 was identified to be caused by CVA6 [24]. Considering that the population of the present study was restricted temporally and geographically, prolonged surveillance and more detailed molecular-typing surveillance of HEVs are needed to better understand the composite viral etiology of HFMD in this area.

Our understanding of epidemiological and genetic characteristics of HEV in the healthy population remains far from complete. In recent years, several studies among healthy individuals in different countries have shown diversity in HEV positive rates. Studies conducted among children in Shenzhen, China [29] and Norway [10,30,31] reported a HEV positive rate of 10.6 and $11.6 \%$ from stool samples respectively. Isolation rates of $64 \%$ and $35 \%$ were obtained from stool samples collected in children under 10-year-old and adults over 21-year-old in Mongolia [32]. With regard to EV71, diversity in positive rate has also been reported among healthy individuals in different countries. Han et al. reported positive rates of $0,4.93$ and $10.29 \%$ in throat swabs and 2.86, 3.94 and $8.82 \%$ in stools from three villages with different HFMD prevalence rate [9]. Studies conducted among children in Finland [33] and Norway $[10,30,31]$ reported an EV71 positive rate of 0.3 and 1.4\% from stool samples respectively, and isolation rate of $1.8 \%$ from stool samples were reported among children in Shenzhen, China [29]. Herein we demonstrated that $23.2 \%$ and $15.0 \%$ out of 254 healthy children carried HEV and EV71 respectively. This might represent the highest EV71 detection rate reported this far among healthy children. Our survey was performed during the local HFMD epidemic period ranging from May to August, which could partially explain the high EV71 frequency. The differences in specimens sampled, selection of subjects, and virus identification methods, as well as the climate, geography, crowding, and socio-economic status factors could also lead to the differences in the positive rate between our study and others.

EV71 is classified into three genotypes A, B and C, and within the genotypes $B$ and $C$, there are further subgenotypes, B1-B5 and C1-C5. Recently, several studies also proposed that subgenotype $\mathrm{C} 4$ strains should be designated as a new genotype $\mathrm{D}$ and that the $\mathrm{B} 5$ isolates to be 


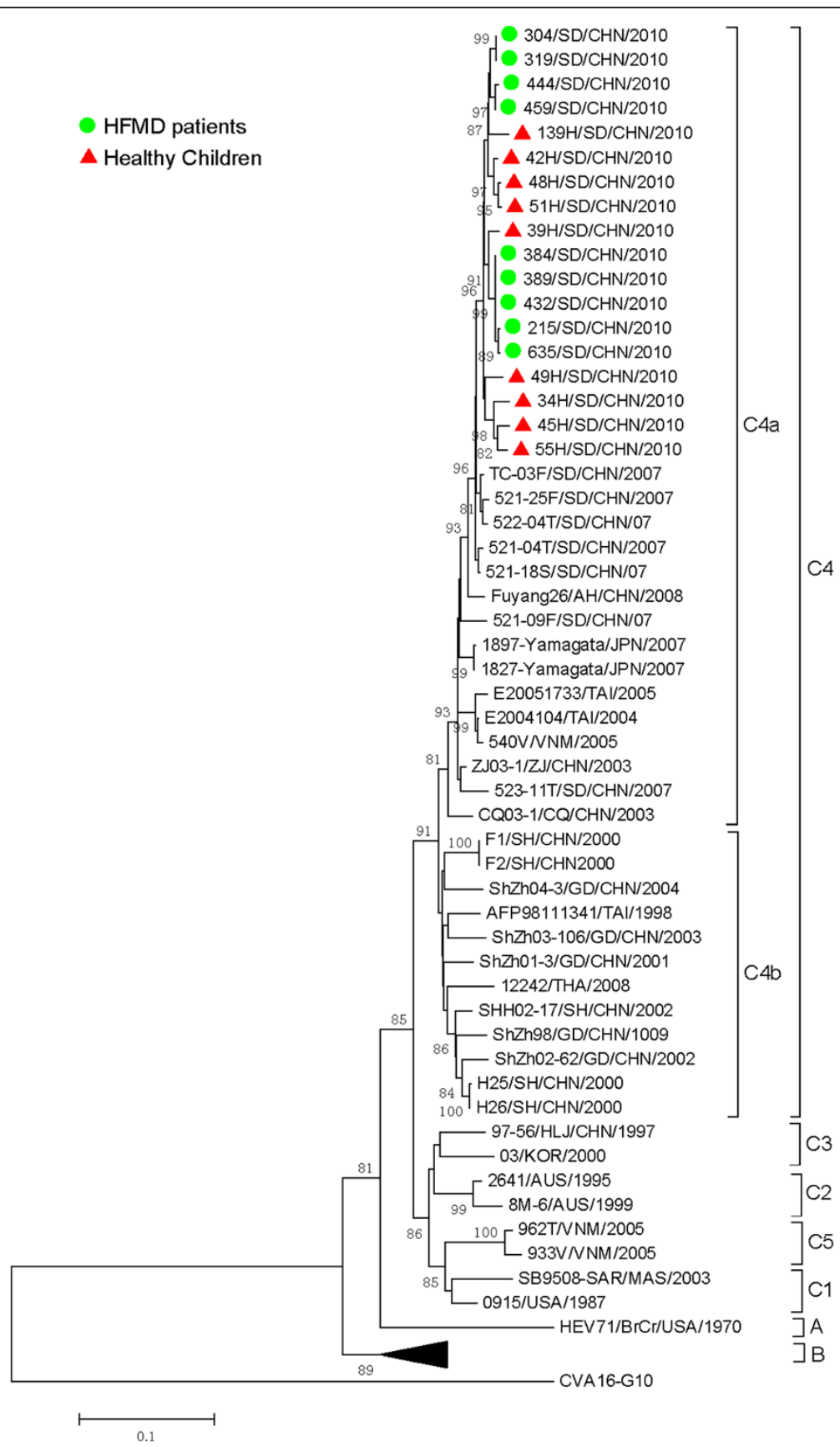

Figure 1 Phylogenetic trees were constructed from the VP1 nucleotide sequences of EV71 using neighbor-joining method with 1000 bootstrap by CLC genomics workbench. The tree was based on the 891 VP1 nucleotide sequences of EV71. The strains labeled with green dots were obtained from healthy children in our study. The strains labeled with red dots were obtained from HFMD patients in our study.

re-designated as B4 [34,35]. In China, the C4 subgenotype was identified as the most prominent circulating EV71 subgenotype [27]. All EV71 sequences reported in this study, regardless of disease status, cluster within the C4a clade, implying that C4a EV71strains predominated in Jining city in 2010. The large numbers of asymptomatic persons who carry HEVs, especially EV71, may serve as a reservoir for transmission of HEV to children and contribute to the large epidemics that occur annually.

Transmission of HEVs within a household is common. A recent prospective family cohort study investigated EV71 patients at a children's hospital and family members of these patients who had EV71 has reported that the EV71 transmission rate to household contacts was 52\%, 
and the transmission rate from children to parents was $41 \%$ [36]. Kuramitsu et al. found that interfamilial spread was responsible for $54 \%$ of non-polio HEV infections in healthy persons [32]. Our family study showed that $18.5 \%$ of family members were positive for HEV, and children had significantly higher HEV positive rate than adults. Limited transmission between parents and children was demonstrated, despite the inconsistent positive detection and incongruent serotypes obtained. Possible transmission between siblings and between husband and wife was suggested, however, only in few families. The information obtained in this study further supported the potential transmission of HEVs even among asymptomatic children, finally leading to a high reservoir for future epidemic. Our study findings stress the importance of personal hygiene to prevent infection with HEVs in the home environment. However, because of the limited number of families, further studies of larger sample size are needed. In addition, prospective follow-up of households would help clarify the distribution and transmission of HEVs within a household.

\section{Conclusions}

In conclusion, this study demonstrated the co-circulation of multiple HEV serotypes in both HFMD cases and healthy children during HFMD epidemics. This study provides useful epidemiological data on the features of the spread of HEV among families as well. These findings have important public health implication for HFMD control, especially in HFMD high epidemic regions.

\section{Abbreviations}

EV: Enteroviruses; HEVs: Human enteroviruses; HFMD: Hand, foot, and mouth disease; EV71: Enterovirus 71; CVA16: Coxsackievirus A16; RT-PCR: Reverse transcription polymerase chain reaction; E: Echovirus.

\section{Competing interests}

The authors have no conflicts of interest to disclose.

\begin{abstract}
Authors' contributions
XAZ and HYW conceived and designed the experiments, performed the experiments, analyzed the data, and drafted the manuscript. XJW and SJD contributed to sample collection. XAZ, XDC, HYW, YW, LYW and DDH contributed to sample preparation and viral detection. WL and CCW conceived and designed the experiments, and drafted and revised the manuscript. XAZ and HYW contributed equally to this work. All authors read and approved the final manuscript.
\end{abstract}

\section{Authors' information}

Dr Zhang is an epidemiologist at the State Key Laboratory of Pathogen and Biosecurity, Beijing Institute of Microbiology and Epidemiology, Beijing, China. Her primary research interests include microbiology, epidemiology, and the genetic susceptibility to infectious diseases such enterovirus 71 infection and influenza.

\section{Acknowledgments}

We thank all the tested individuals, their families, and collaborating clinicians for their participation.

This study has neither been presented nor submitted or accepted anywhere.

\section{Financial support}

This work was supported by grants from the China Mega-Project on Infectious Disease Prevention (No. 2013ZX10004202-002), National Natural Science Foundation (No. 81222037, 81202253) and Shandong Scientific and Technical Supporting Program (No. 2009GG10002055).

\section{Author details}

${ }^{1}$ Graduate School of Anhui Medical University, 230032, Hefei, P. R. China.

${ }^{2}$ State Key Laboratory of Pathogen and Biosecurity, Beijing Institute of Microbiology and Epidemiology, 20 Dong-Da Street, Fengtai District, Beijing 100071, P. R. China. ${ }^{3}$ Shandong Provincial Center for Disease Control and Prevention, 250001, Jinan, P. R. China.

Received: 1 October 2013 Accepted: 26 December 2013

Published: 27 December 2013

\section{References}

1. Solomon T, Lewthwaite P, Perera D, Cardosa MJ, McMinn P, Ooi MH: Virology, epidemiology, pathogenesis, and control of enterovirus 71. Lancet Infect Dis 2010, 10(11):778-790.

2. Yip CC, Lau SK, Woo PC, Yuen KY: Human enterovirus 71 epidemics: what's next? Emerg Health Threats J 2013, 6:19780.

3. Zhang Y, Tan XJ, Wang HY, Yan DM, Zhu SL, Wang DY, Ji F, Wang XJ, Gao $Y J$, Chen L, et al: An outbreak of hand, foot, and mouth disease associated with subgenotype C4 of human enterovirus 71 in Shandong, China. J Clin Virol 2009, 44(4):262-267.

4. Yang F, Ren L, Xiong Z, Li J, Xiao Y, Zhao R, He Y, Bu G, Zhou S, Wang J, et al: Enterovirus 71 outbreak in the People's Republic of China in 2008. J Clin Microbiol 2009, 47(7):2351-2352.

5. He SJ, Han JF, Ding XX, Wang YD, Qin CF: Characterization of enterovirus 71 and coxsackievirus A16 isolated in hand, foot, and mouth disease patients in Guangdong, 2010. Int J Infect Dis 2013, 17(11):e1025-1030.

6. Lu QB, Zhang XA, Wo Y, Xu HM, Li XJ, Wang XJ, Ding SJ, Chen XD, He C, Liu LJ, et al: Circulation of Coxsackievirus A10 and A6 in hand-foot-mouth disease in China, 2009-2011. PLoS One 2012, 7(12):e52073.

7. Yang F, Zhang T, Hu Y, Wang X, Du J, Li Y, Sun S, Sun X, Li Z, Jin Q: Survey of enterovirus infections from hand, foot and mouth disease outbreak in China, 2009. Virol J 2011, 8:508.

8. Tseng FC, Huang HC, Chi CY, Lin TL, Liu CC, Jian JW, Hsu LC, Wu HS, Yang $J Y$, Chang YW, et al: Epidemiological survey of enterovirus infections occurring in Taiwan between 2000 and 2005: analysis of sentinel physician surveillance data. J Med Virol 2007, 79(12):1850-1860.

9. Han J, Ma XJ, Xu WB, Wan JF, Li Q, Tian C, Gao C, Wang M, Tang LY, Zhang $Y$, et al: EV71 viral secretion by symptomatic hand foot and mouth disease patients and their asymptomatic close contacts. J Infect 2011, 62(1):107-108.

10. Witso E, Palacios G, Cinek O, Stene LC, Grinde B, Janowitz D, Lipkin WI, Ronningen KS: High prevalence of human enterovirus a infections in natural circulation of human enteroviruses. J Clin Microbiol 2006, 44(11):4095-4100

11. Simonen-Tikka ML, Hiekka AK, Klemola P, Poussa T, Ludvigsson J, Korpela R, Vaarala $\mathrm{O}$, Roivainen M: Early human enterovirus infections in healthy Swedish children participating in the PRODIA pilot study. J Med Virol 2012, 84(6):923-930.

12. Verstrepen WA, Bruynseels $P$, Mertens AH: Evaluation of a rapid real-time RT-PCR assay for detection of enterovirus RNA in cerebrospinal fluid specimens. J Clin Virol 2002, 25(Suppl 1):S39-S43.

13. Nix WA, Oberste MS, Pallansch MA: Sensitive, seminested PCR amplification of VP1 sequences for direct identification of all enterovirus serotypes from original clinical specimens. J Clin Microbiol 2006, 44(8):2698-2704.

14. Xu M, Su L, Cao L, Zhong H, Dong N, Xu J: Enterovirus genotypes causing hand foot and mouth disease in Shanghai, China: a molecular epidemiological analysis. BMC Infect Dis 2013, 13(1):489.

15. Zhang L, Chen QY, Liu H, Tang LQ, Mai HQ: Emerging treatment options for nasopharyngeal carcinoma. Drug Des Devel Ther 2013, 7:37-52.

16. He YQ, Chen L, Xu WB, Yang H, Wang HZ, Zong WP, Xian HX, Chen HL, Yao $X J, H u Z L$, et al: Emergence, circulation, and spatiotemporal phylogenetic analysis of coxsackievirus a6- and coxsackievirus a10-associated hand, foot, and mouth disease infections from 2008 to 2012 in Shenzhen, China. J Clin Microbiol 2013, 51(11):3560-3566. 
17. Cabrerizo M, Tarrago D, Munoz-Almagro C, Del Amo E, Dominguez-Gil M, Eiros JM, Lopez-Miragaya I, Perez C, Reina J, Otero A, et al: Molecular epidemiology of enterovirus 71, coxsackievirus A16 and A6 associated with hand, foot and mouth disease in Spain. Int J Infect Dis 2013. in press

18. Park SH, Choi SS, Oh SA, Kim CK, Cho SJ, Lee JH, Ryu SH, Pak SH, Jung SK, Lee Il, et al: Detection and characterization of enterovirus associated with herpangina and hand, foot, and mouth disease in Seoul, Korea. Clin Lab 2011, 57(11-12):959-967.

19. Gopalkrishna V, Patil PR, Patil GP, Chitambar SD: Circulation of multiple enterovirus serotypes causing hand, foot and mouth disease in India. J Med Microbiol 2012, 61(Pt 3):420-425.

20. Wu Y, Yeo A, Phoon MC, Tan EL, Poh CL, Quak SH, Chow VT: The largest outbreak of hand; foot and mouth disease in Singapore in 2008: the role of enterovirus 71 and coxsackievirus A strains. Int J Infect Dis 2010, 14(12):e1076-e1081.

21. Fujimoto T, lizuka S, Enomoto M, Abe K, Yamashita K, Hanaoka N, Okabe N, Yoshida H, Yasui Y, Kobayashi M, et al: Hand, foot, and mouth disease caused by coxsackievirus A6, Japan, 2011. Emerg Infect Dis 2012, 18(2):337-339.

22. Montes M, Artieda J, Pineiro LD, Gastesi M, Diez-Nieves I, Cilla G: Hand, foot, and mouth disease outbreak and coxsackievirus A6, northern Spain, 2011. Emerg Infect Dis 2013, 19(4):676-678.

23. Flett K, Youngster I, Huang J, McAdam A, Sandora TJ, Rennick M, Smole S, Rogers SL, Nix WA, Oberste MS, et al: Hand, foot, and mouth disease caused by coxsackievirus a6. Emerg Infect Dis 2012, 18(10):1702-1704

24. Puenpa J, Chieochansin T, Linsuwanon P, Korkong S, Thongkomplew S, Vichaiwattana P, Theamboonlers A, Poovorawan Y: Hand, foot, and mouth disease caused by coxsackievirus A6, Thailand, 2012. Emerg Infect Dis 2013, 19(4):641-643.

25. Huang WC, Huang LM, Lu CY, Cheng AL, Chang LY: Atypical hand-footmouth disease in children: a hospital-based prospective cohort study. Virol J 2013, 10:209.

26. Chang LY, Lin TY, Huang YC, Tsao KC, Shih SR, Kuo ML, Ning HC, Chung PW Kang CM: Comparison of enterovirus 71 and coxsackie-virus A16 clinical illnesses during the Taiwan enterovirus epidemic, 1998. Pediatr Infect Dis J 1999, 18(12):1092-1096.

27. Tan X, Huang X, Zhu S, Chen H, Yu Q, Wang H, Huo X, Zhou J, Wu Y, Yan D, et al: The persistent circulation of enterovirus 71 in People's Republic of China: causing emerging nationwide epidemics since 2008. PLoS One 2011, 6(9):e25662.

28. Yang F, Du J, Hu Y, Wang X, Xue Y, Dong J, Sun L, Li Z, Li Y, Sun S, et al: Enterovirus coinfection during an outbreak of hand, foot, and mouth disease in Shandong, China. Clin Infect Dis 2011, 53(4):400-401.

29. Wu W, Xu WB, Chen L, Chen HL, Liu Q, Wang DL, Chen YJ, Yao W, Li G, Feng $B$, et al: Molecular identification and analysis of human enteroviruses isolated from healthy children in shenzhen, china from 2010 to 2011. PLoS One 2013, 8(6):e64889.

30. Witso E, Palacios G, Ronningen KS, Cinek O, Janowitz D, Rewers M, Grinde B, Lipkin WI: Asymptomatic circulation of HEV71 in Norway. Virus Res 2007, 123(1):19-29.

31. Cinek $O$, Witso $E$, Jeansson $S$, Rasmussen $T$, Drevinek $P$, Wetlesen $T$, Vavrinec J, Grinde B, Ronningen KS: Longitudinal observation of enterovirus and adenovirus in stool samples from Norwegian infants with the highest genetic risk of type 1 diabetes. J Clin Virol 2006, 35(1):33-40.

32. Kuramitsu M, Kuroiwa C, Yoshida H, Miyoshi M, Okumura J, Shimizu H, Narantuya L, Bat-Ochir D: Non-polio enterovirus isolation among families in Ulaanbaatar and Tov province, Mongolia: prevalence, intrafamilial spread, and risk factors for infection. Epidemiol Infect 2005, 133(6):1131-1142.

33. Honkanen $\mathrm{H}$, Oikarinen $\mathrm{S}$, Pakkanen O, Ruokoranta T, Pulkki MM, Laitinen $\mathrm{OH}$, Tauriainen S, Korpela S, Lappalainen M, Vuorinen T, et al: Human enterovirus 71 strains in the background population and in hospital patients in Finland. J Clin Virol 2013, 56(4):348-353.

34. Li W, Yi L, Su J, Lu J, Zeng H, Guan D, Ma C, Zhang W, Xiao H, Li H, et al: Seroepidemiology of human enterovirus71 and coxsackievirusA16 among children in Guangdong province, China. BMC Infect Dis 2013, 13:322.

35. Yip CC, Lau SK, Lo JY, Chan KH, Woo PC, Yuen KY: Genetic characterization of EV71 isolates from 2004 to 2010 reveals predominance and persistent circulation of the newly proposed genotype $D$ and recent emergence of a distinct lineage of subgenotype C2 in Hong Kong. Virol J 2013, 10:222.

36. Chang LY, Tsao KC, Hsia SH, Shih SR, Huang CG, Chan WK, Hsu KH, Fang TY, Huang YC, Lin TY: Transmission and clinical features of enterovirus 71 infections in household contacts in Taiwan. JAMA 2004, 291(2):222-227

doi:10.1186/1471-2334-13-606

Cite this article as: Zhang et al:: Prevalence of enteroviruses in children with and without hand, foot, and mouth disease in China. BMC Infectious Diseases 2013 13:606

\section{Submit your next manuscript to BioMed Central and take full advantage of:}

- Convenient online submission

- Thorough peer review

- No space constraints or color figure charges

- Immediate publication on acceptance

- Inclusion in PubMed, CAS, Scopus and Google Scholar

- Research which is freely available for redistribution

Submit your manuscript at www.biomedcentral.com/submit
C) Biomed Central 